\title{
Effect of Blade Coating on a Centrifugal Pump Operation under Sediment-Laden Water Flow
}

\author{
Yong Wang 1 - Zilong Zhang ${ }^{1}$ - Jie Chen ${ }^{2}$ - Houlin Liu 1 - Xiang Zhang3,* - Marko Hočevar ${ }^{4}$ \\ 1 Jiangsu University, Research Center of Fluid Machinery Engineering and Technology, China \\ 2 Beijing Institute of Technology, School of Mechanical and Vehicular Engineering, China \\ ${ }^{3}$ Xihua University, Key Laboratory of Fluid and Power Machinery, China \\ ${ }^{4}$ University of Ljubljana, Faculty of Mechanical Engineering, Slovenia
}

\begin{abstract}
Applying a high strength coating on a blade's surface could significantly prolong the service life of a centrifugal pump under sediment-laden water flow because of its protection. To explore the effect of blade coating, the characteristics of energy, vibration and pressure fluctuation of a centrifugal pump (the specific speed $\left(n_{s}\right)$ is 81.46 ) with different polyurethane coating thickness coefficients were experimentally studied under sediment-laden water flow. Keeping the blade outlet angle, blade inlet angle and blade shape unchanged, the head $\mathrm{H}$ and efficiency $\eta$ under both sediment-laden flow and clear water flow decrease significantly as the coating thickness coefficient increases. The axis rotating frequency and blade passing frequency are the main excitation frequencies of the pump vibration velocity amplitude and outlet pressure fluctuation. The vibration velocity amplitude and outlet pressure fluctuation at the frequency of $1 \mathrm{BPF}$ are the largest. At the frequency of 1 axis rotating frequency, they are the second in all cases. The peak values of both vibration velocity amplitude and outlet pressure fluctuation are proportional to the coating thickness coefficient. An analysis was performed for several increasing coating thicknesses, corresponding to coating coefficients from $K_{0}$ to $K_{3}$. When the coating thickness coefficients are $K_{0}, K_{1}$, and $K_{2}$, the peak value of vibration velocity amplitude under sediment-laden flow is larger than that under clear water flow, but the very small difference between them undercoating thickness coefficient $\mathrm{K}_{3}$. The peak values of pressure fluctuations under different flow rates decrease first and then increase with the increasing coating thickness coefficient, and lowest points are all located at the coating thickness coefficient $K_{1}$.
\end{abstract}

Keywords: blade coating, vibration, pressure fluctuation, sediment-laden flow, centrifugal pump

\section{Highlights}

- The characteristics of energy, vibration and pressure fluctuation of a blade-coated centrifugal pump under sediment-laden flow are studied experimentally.

- The blade coating has a small negative effect on the pump energy characteristics, although it may prevent surface wear.

- The peak values of vibration velocity amplitude and pressure fluctuation are all located at the same frequency in all experimental cases.

- When the flow rate is small, the peak values of vibration velocity amplitude under sediment-laden flow are lower than that under clear water flow.

- Under both flows, the peak values of pressure fluctuation decrease first and then increase with the increasing coating thickness coefficient.

\section{INTRODUCTION}

As essential equipment for fluid transportation, the centrifugal pump plays an important role in irrigation, metallurgy, water conservation, and mining industry, etc. The working medium of the pump is sedimentladen flow rather than a single medium water flow. The solid phase of sediment-laden flow can abrade the wet parts, causing noise, vibration, leakage and, finally, pump breakdown. Due to the high rotational speed of the impeller, breakage is most likely to happen on the pressure surface of the impeller blades in a solidliquid two-phase flow centrifugal pump. Furthermore, the strong vibration, pressure fluctuation and abrasion of a damaged centrifugal pump significantly reduces the reliability of the pumping station system and causes environmental noise pollution. Therefore, it is important to improve the performance of the pump when transporting solid-liquid two-phase liquid.

At present, scholars have done much research on the energy characteristics, pressure fluctuation, and vibration for some typical hydraulic machinery under solid-liquid two-phase flow. Work is performed experimentally and by numerical simulation. Wang and Qian [1] investigated the effects of the silt concentration and grain size on the head and efficiency of the pump. They established a relationship for the head reduction factors as a function of the silt parameters for the double-suction centrifugal pump. Jin [2] numerically carried out the response law of impeller channel and vibration in a centrifugal pump and concluded that changing the number of impeller blades can effectively reduce the vibration intensity and avoid resonance. Rodriguez et al. [3] presented an 
analytical prediction method for the peak frequency and amplitude of the rotor-stator interaction (RSI) in large pump turbines in a qualitative way. Han et al. [4] analysed the pressure fluctuations and the axial force on the impeller in a solid-liquid two-phase flow centrifugal pump. In this study, a three-dimensional simulation was performed for the solid-liquid twophase turbulent flow in a centrifugal pump with a radial diffuser by the mixture multiphase model, the large eddy simulation turbulence model, and sliding mesh technology. Kumar et al. [5] evaluated the centrifugal slurry pump total head, efficiency, and input power operating with a multi-sized particulate slurry of bottom ash and fly ash mixtures at different flow rates. Zhao et al. [6] numerically simulated the solid-liquid two-phase flow of a double suction centrifugal pump with the computational fluid dynamics. The path of the particle in the channel under different particle sizes and particle volume concentrations was revealed. Hazra and Steiner [7] computed the flow field of diluting two-phase flow pump in turbulent conditions for the continuous phase with the Reynolds averaged Navier-Stokes equations together in a mixing length turbulence modelling. The large-size particles accumulated at the lower pressure zone near the cashing wall or the rotating shaft. Zhang et al. [8] numerically simulated and analysed turbulent solid-liquid two-phase flows in a low-specific-speed centrifugal pump, in which the influences of rotation and curvature were fully taken into account; the obtained results preliminarily reveal the characteristics of solid-liquid two-phase flow in a centrifugal pump. Tse and Wang [9] designed an efficient prognostic method to assess the performance degradation of a pump though extracting statistical features of vibration signals from on-site operating slurry pumps. This method has better prediction accuracy when compared to other procedures. Therefore, the research on the energy characteristics, vibration, and pressure fluctuation of a solid-liquid two-phase flow centrifugal pump has great significance.

At the same time, to improve the performance of the pump when they work under sediment-laden flow, researchers have found that spraying the anti-wear coating on the surface of wet parts is a good form of protection. Generally, coating material with better wear resistance, including epoxy resin mortar coatings [10] and [11], composite nylon coatings [12], polyurethane coatings [13] and other polymer coatings [14], alleviates the problem of the wear and tear of solidliquid two-phase flow pumps. Luo et al. [15] sprayed a polyurethane coating on the surface of wet parts of a solid-liquid two-phase flow centrifugal pump and explored the performance of the centrifugal pump with different coating thickness coefficients under a water medium. He also measured internal flow, pressure fluctuations and radial force of the model pump and compared it with numerical simulation, which showed some similarity. Serrano et al. [16] analysed the wear volume of the impeller blade caused by the relative velocity of the mixture (water and sediment-laden) and the wear accumulated in an annual hydrological cycle was estimated mathematically. Tarodiya and Gandhi [17] experimentally and numerically investigated the performance of a centrifugal slurry pump and presented a numerical modelling approach to predict the performance and wear characteristics of the pump. Walker and Robbie [18] performed laboratory wear tests to measure the wear resistance of natural rubber and eutectic and hypereutectic white iron under abrasion conditions in a centrifugal slurry pump. Zhang [19] had shown that elastic polyurethane coating could reduce erosion wear damage caused by the sediment-laden flow on hydraulic machines operating at high speed, which could extend the service life of the pump impellers and turbine blades.

It can be seen from the above studies that the energy, vibration, and pressure fluctuation characteristics of the pump under sediment-laden flow are very different from those under the clear water flow medium. For both pressure pulsation and vibration, the greater the peak value, the greater the impact power on the device, and the higher the peak frequency, the higher the impact times on the device per unit time. After working for a long time, the small difference of vibration and pulsation may cause serious mechanical failures, such as bearing wear and shaft deformation. There are few studies on solid-liquid two-phase flow centrifugal pumps with protective coatings on blades. At present, the research in this area is mainly carried out through numerical simulations. Although numerical simulation is an effective method for predicting outcomes, accurate experiments are essential in research.

Therefore, a polyurethane coating with excellent wear resistance was applied on the blade pressure surface of a centrifugal pump, where the surface is most easily worn. The effect of the blade coating thickness coefficient on the energy, vibration and pressure fluctuation of a centrifugal pump operation under sediment-laden water flow was experimentally studied. 


\section{RESEARCH OBJECTIVE AND TEST SYSTEM}

\subsection{Selection of Coating Material}

Applying protected coating on the blade can significantly improve the life of the pump. Our research group experimentally studied the abrasion resistance and cavitation erosion resistance of three materials (epoxy resin mortar, composite resin mortar and polyurethane), which are frequently used for the turbine blade as a protected coat, and concluded that polyurethane shows good performance [20]. Polyurethane coating has also been confirmed by Zhou et al. [21] and Zhong et al. [22], showing that it has numerous advantages including abrasion and cavitation resistance using in wet surface protection.

Therefore, polyurethane (curing agent, catalyst, stainless steel flake powder, weight ratio of acetophenone to acetone is $1: 0.11: 0.015: 0.4: 1.25$ : 1.25) was selected as the protective material used on the pressure surface of the blades and using 3D printing technology to make a mould to keep the shape and uniform, pouring the blade coating, and finally drying by airing.

\subsection{The Model Pump}

The single-stage single-suction horizontal centrifugal model pump with straight blades was used in the experiment. The design parameters of the model pump are as follows: design flow rate $Q_{d}=20 \mathrm{~m}^{3} / \mathrm{h}$, head $H=22 \mathrm{~m}$, efficiency $\eta=48 \%$, rated shaft power $P=1.5 \mathrm{~kW}$, rotation speed $n=2900 \mathrm{rpm}$, and specific speed $n_{s}=81.46$. The main geometric parameters of the model pump are shown in Table 1.

Table 1. Main parameters of the model pump

\begin{tabular}{llcc}
\hline \multirow{4}{*}{ Impeller } & Inlet diameter [mm] & $D_{1}$ & 65 \\
\cline { 2 - 4 } & External diameter [mm] & $D_{2}$ & 160 \\
\cline { 2 - 4 } & Width of outlet [mm] & $b_{2}$ & 7 \\
\cline { 2 - 4 } & Blade thickness [mm] & $\delta$ & 6 \\
\cline { 2 - 4 } Volute & Number of blades & $z$ & 3 \\
\hline & Inlet diameter [mm] & $D_{3}$ & 182 \\
\cline { 2 - 4 } & Inlet width [mm] & $b_{3}$ & 26 \\
\cline { 2 - 4 } & Outlet diameter [mm] & $D_{4}$ & 50 \\
\hline
\end{tabular}

\subsection{Experimental Test System}

The schematics of the measuring station are shown in Fig. 1. The measuring station consists of piping, a water tank, outlet valve, a submersible pump, inlet valve, an electromagnetic flowmeter, a model pump with variable-frequency drive, a pressure fluctuation measuring system, a vibration measuring system, and an electric power measuring system.

Irrigation water, especially in China, features the diameter of particles in sediment-laden flow around $0.03 \mathrm{~mm}$ and an average bulk concentration of about $3 \%$ [23]. Therefore, sediment-laden water under these features is selected as a working medium. A submersible pump is set in the water storage tank to prevent particles in sediment-laden water from settling, thus keeping the sediment-laden particle concentration in the pump during the test constant. The main parameters of submersible pump are as follows: design flow $Q_{d}=25 \mathrm{~m}^{3} / \mathrm{h}$, head $H=18 \mathrm{~m}$, rated shaft power $P=1.5 \mathrm{~kW}$, rotational speed $n=1450 \mathrm{r} / \mathrm{min}$, and specific speed $n_{s}=50.5$. The experimental test system is shown in Fig. 1.

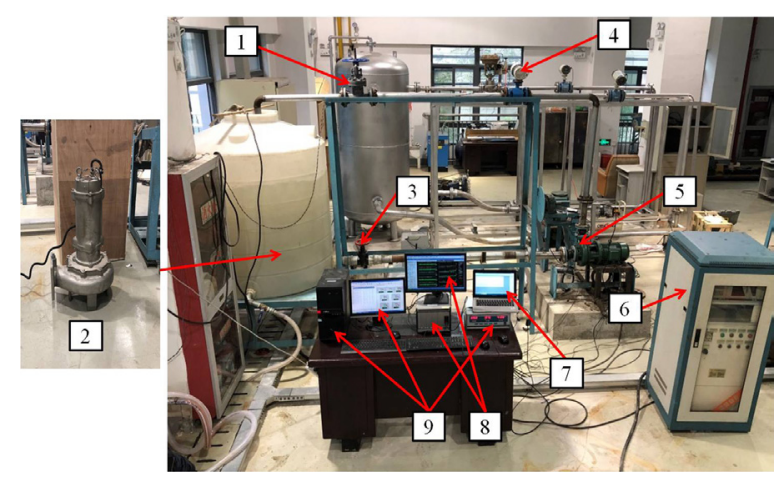

Fig. 1. Test system; 1) outlet valve; 2) submersible pump; 3) inlet valve; 4) electromagnetic flowmeter; 5) model pump; 6) variable-frequency drive; 7) pressure fluctuation measuring system; 8) vibration measuring system; 9) energy performance measuring system

The energy generated by vibration is directly related to the vibration velocity. By using the integral function in DASP (data acquisition and signal processing) software, the vibration acceleration is translated to the vibration velocity. The time-domain of the vibration signal is converted into the frequency domain diagram by using the fast Fourier transform (FFT) method. The vibration velocity (root mean square of velocity) is applied to evaluate the overall vibration. The vibration velocity is measured with an INV9832 acceleration sensor (frequency interval from $0.5 \mathrm{kHz}$ to $8 \mathrm{kHz}$, range of \pm 50 times the acceleration of gravity, measurement error $\pm 0.10 \%$ ).

To measure vibration, sensors are located at the following locations: pump flange inlet, pump body, pump flange outlet and electric motor pedestal, as shown in Table 2 and Fig. 2. 
Table 2. Measurement locations for vibration measurements

\begin{tabular}{cccc}
\hline Point $M_{1}$ & Point $M_{2}$ & Point $M_{3}$ & Point $M_{4}$ \\
\hline Flange inlet & Pump body & Flange outlet & Electric motor pedestal \\
\hline
\end{tabular}

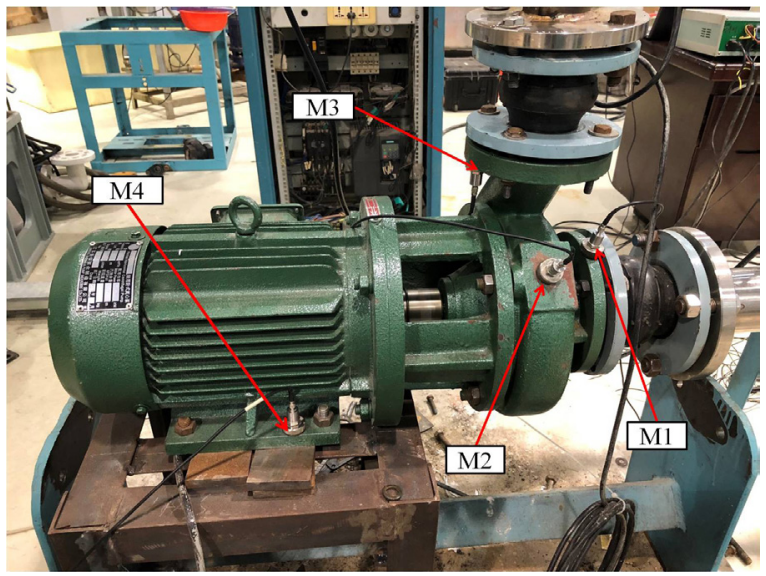

Fig. 2. Measurement locations for vibration test

The FFT is used to transform the pressure fluctuation time domain signal to the frequency domain. Dimensionless representation of pressure fluctuations is defined as follows:

$$
C_{p}=\frac{\sqrt{\left(\frac{1}{N}\right)\left(p_{i}-\bar{p}\right)^{2}}}{0.5 \rho u_{2}^{2}} .
$$

Here, pressure fluctuation coefficient $C_{p}$ is the root mean square value of the pressure fluctuation data measured at the measurement locations, divided by dynamic pressure. $\bar{p}$ and $p_{i}$ are the average static pressure and fluctuating static pressure, respectively, while the unit is $\mathrm{Pa} . \rho$ is the medium density, the unit is $\mathrm{kg} / \mathrm{m}^{3} . N$ is the sample number. $u_{2}$ is the impeller outlet circumferential speed, and the unit is $\mathrm{m} / \mathrm{s}$.

The pressure fluctuation is measured with a CY301 intelligent digital pressure sensor (maximum frequency of $1 \mathrm{kHz}$, maximum absolute pressure of $300 \mathrm{kPa}$, measurement error $\pm 0.10 \%$ ). The data acquisition and processing system for pressure fluctuations is shown in Fig. 3.

The measurement location of pump outlet pressure fluctuation is set at $4 \mathrm{D}$ downstream pipe diameter $D$ distance from the flange. The pump head is the energy increase of liquid from the inlet to outlet of the pump generated by rotational impeller. The measurement locations of pump inlet and outlet pressure are set at 2 times the upstream and downstream pipe diameter distance from the flange, respectively. Locations of pressure measurements are shown in Fig. 4.

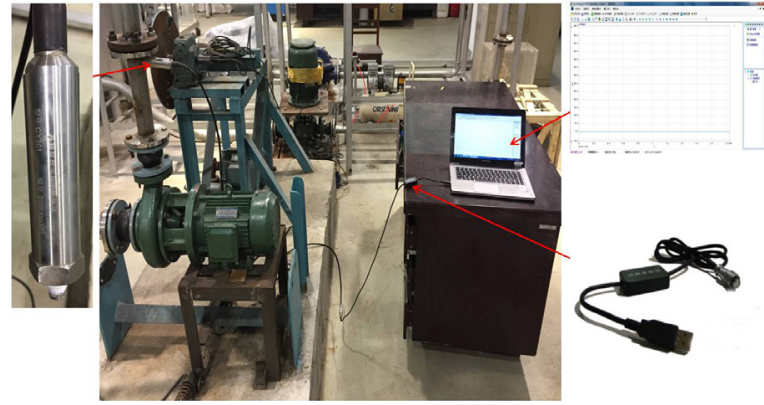

Fig. 3. Data acquisition and processing system for pressure fluctuations

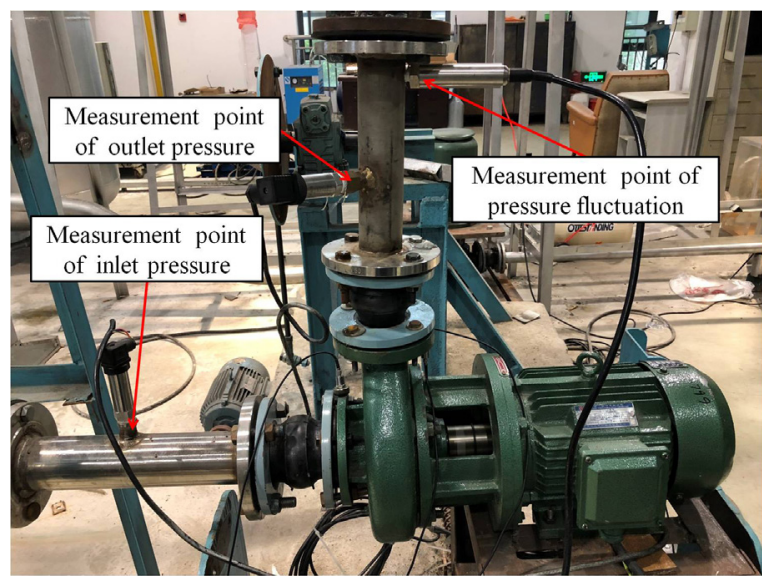

Fig. 4. Measurement locations for pressure fluctuations

Also, the flow is pumped from and returned to a $10 \mathrm{~m}^{3}$ tank. The rotational speed is measured by a speed sensor AR926 (rotation from $2.5 \mathrm{rpm}$ to $10^{5}$ $\mathrm{rpm}$, measurement error $\pm 0.05 \%$ ). The shaft torque is measured by a torque sensor of model CYT-302 (maximum torque $200 \mathrm{Nm}$, measurement error \pm 0.3 $\%$ ). The static pressure is measured by a pressure sensor MIK-P300 (maximum static pressure of 1 $\mathrm{MPa}$, measurement error $\pm 0.1 \%$ ). The flow rate is measured by a magnetic flow meter KEFN (maximum flow rate of $100 \mathrm{~m}^{3} / \mathrm{h}$, measurement error $\pm 0.1 \%$ ). The collected data are analysed and processed when the test system is stable after a period of operation to ensure the validity of data.

\subsection{Dimensionless Coating Thickness}

We have used the coating thickness in its dimensionless form, as follows:

$$
K_{i}=\frac{6 \delta_{i}}{\delta}
$$


Here, $K_{i}$ is the coating thickness coefficient, $i$ is taken as $0,1,2,3 ; \delta_{i}$ is the coating thickness, unit is $\mathrm{mm} ; \delta$ is the blade thickness, in our case $6 \mathrm{~mm}$.

The coating thicknesses $\delta_{i}$ are set as $0 \mathrm{~mm}, 1 \mathrm{~mm}$, $2 \mathrm{~mm}, 3 \mathrm{~mm}$, and the corresponding coating thickness coefficients are $K_{0}=0, K_{1}=1, K_{2}=2$, and $K_{3}=3$, respectively. The impellers with different coating thickness coefficients are shown in Fig. 5.

a)
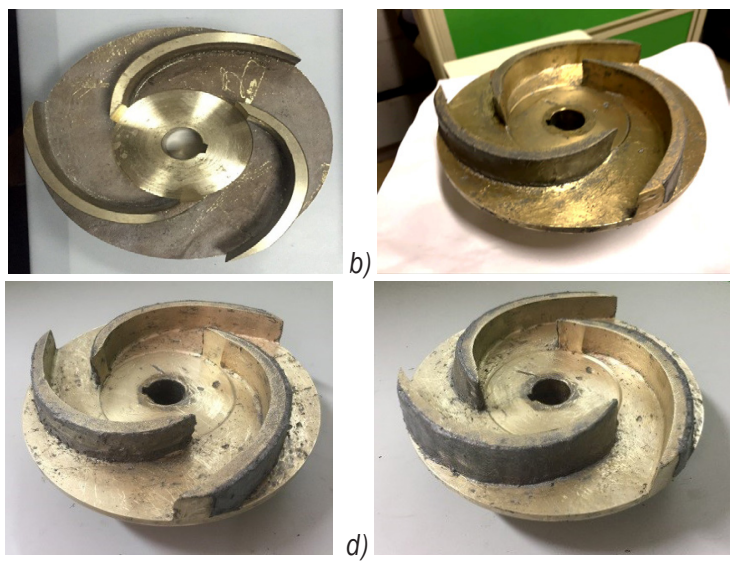

d)

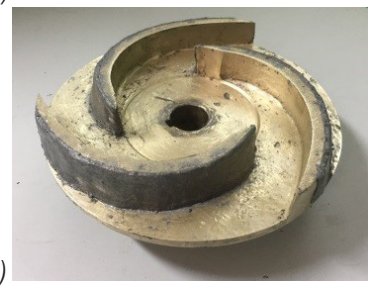

Fig. 5. Impellers with different coating thickness coefficients;

a) $K_{0}$; b) $K_{1}$, c) $K_{2}$; d) $K_{3}$

\section{RESULTS AND ANALYSIS}

\subsection{Energy Characteristics}

Fig. 6 shows the energy characteristics of the model pump for four coating thickness coefficients under sediment-laden and clear water flow.

Fig. 6a shows that the head under sedimentladen flow is slightly larger than under clear water flow under the same coating thickness coefficient when the volumetric flow rate is below around 0.9 $Q_{\mathrm{d}}$. According to [24], at low volumetric flow rates, this can be explained by the solid particles slightly decreasing disturbances near the wet surface, which reduces the turbulent boundary layer thickness and energy loss. However, the head under the sedimentladen flow decreases faster when the volumetric flow exceeds around $0.9 Q_{\mathrm{d}}$, because the whole velocity field of two-phase flow is more aberrant relative to the clear water flow. The velocity gradient is much greater under the large flow rate, which increases the loss generated by the velocity difference between water and particles. The contribution of this loss is more than the head increase from the disturbance elimination of particle swarm. Thus, the pump head is slightly lower than that under sediment flow under the larger flow rate.

In contrast, the head under both sediment-laden flow and clear water flow decreases significantly as the coating thickness coefficient increases. The theoretical pumping head $H_{t}$ and efficiency $\eta$ are derived from Euler equations, Eqs. (3) to (6) [24] and [25]. Here, $u_{1}, u_{2}, v_{u 1}$ and $v_{u 2}$ are the circumferential components of the inlet and outlet circumferential speed and absolute velocity, respectively; $\beta_{2}$ is blade angle at the exit; $\varphi_{2}$ is extrusion coefficient at impeller outlet; $s_{u 2}$ is blade outlet thickness; $\gamma$ is the angle between the impeller outlet axis line and the streamline; $h_{0}$ is slip coefficient; is the pump efficiency; $\eta_{h}$ is the pump hydraulic efficiency; $\eta_{v}$ is the volumetric efficiency; $\eta_{m}$ is the mechanical efficiency; $P_{0}$ is the power of liquid energy increase; $P_{e}$ is the shaft power;
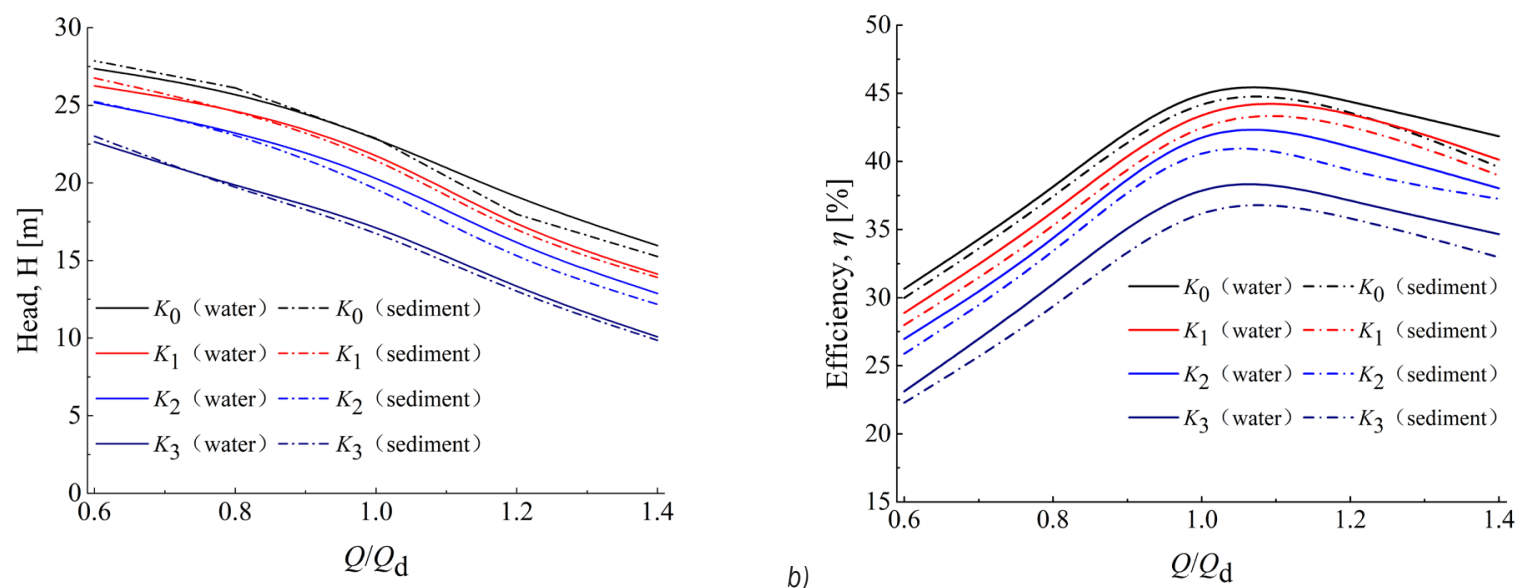

Fig. 6. Energy characteristics for different coating thickness coefficients and working mediums; a) head; b) efficiency 


$$
\begin{gathered}
H_{t}=\frac{u_{2} v_{u 2}-u_{1} v_{u 1}}{g}= \\
=\frac{u_{2}}{g}\left(u_{2} h_{0}-\frac{Q_{d}}{\pi D_{2} b_{2} \varphi_{2} \tan \beta_{2}}\right)-\frac{u_{1} v_{u 1}}{g}, \\
\varphi_{2}=1-\frac{z S_{u 2}}{\pi D_{2}}=1-\frac{z \delta}{\pi D_{2} \sin \gamma \sin \beta_{2}^{\prime}}, \\
h_{0}=1-\frac{\pi \sin \beta_{2}}{z}, \\
\eta=\eta_{h} \eta_{v} \eta_{m}=\frac{P_{0}}{P_{e}} .
\end{gathered}
$$

Based on the formulas mentioned above, as the coating thickness increases, the blade thickness $\delta$ becomes larger. Since the coating is sprayed uniformly, the blade profile is unchanged, that is to say, $D_{2}, \gamma$ and $\beta_{2}$ are not changed. Based on Eq. (3), it concludes that blade coating makes $\delta$ larger while $\varphi_{2}$ becomes smaller. Eq. (5) shows that the slip coefficient $h_{0}$ does not change. Therefore, the blade coating makes $\varphi_{2}$ smaller when other parameters remain unchanged. Based on Eq. (3), the theoretical head of the model pump is reduced; therefore, with the increase of the impeller coating thickness coefficient, the model pump head is gradually reduced.

From Fig. 6b, the efficiency gradually decreases with the increase of the coating thickness coefficient for all flow rates. Based on Eq. (6), the pump efficiency $\eta$ is related directly to the hydraulic efficiency $\eta_{h}$, the volumetric efficiency $\eta_{v}$, and the mechanical efficiency $\eta_{m}$. The hydraulic efficiency $\eta_{h}$ will be the only changed parameter of the three above when the coating thickness coefficient varies. The liquid shock at blade inlet and swirl at the blade outlet will be more significant and make more energy loss under a larger coating thickness coefficient. Therefore, the pump efficiency $\eta$ drops as the coating thickness coefficient increases.

At the same time, the pump efficiency under sediment-laden flow is slightly smaller than that under clear water flow. On the one hand, because of different density, viscosity and physical state, the velocities of solid and liquid at the same point are different, especially in highly turbulent flow, which increases the loss generated by the friction of solidliquid flow. On the other hand, solid particles rush and hit the wet surface frequently, which generates energy loss. Based on Eq. (6), because of these two points

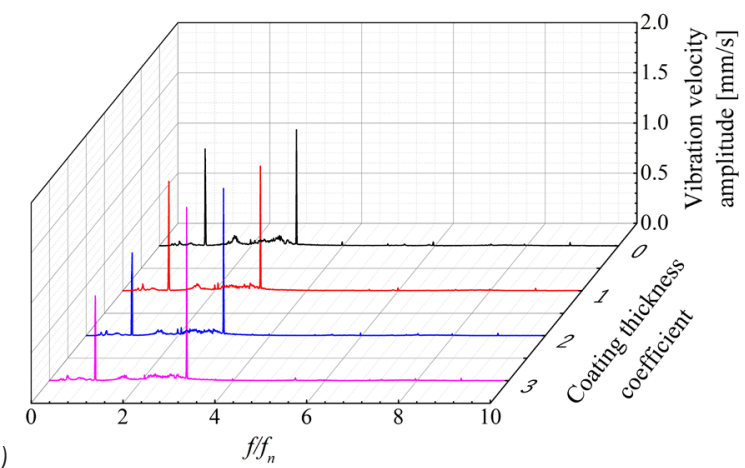

a)

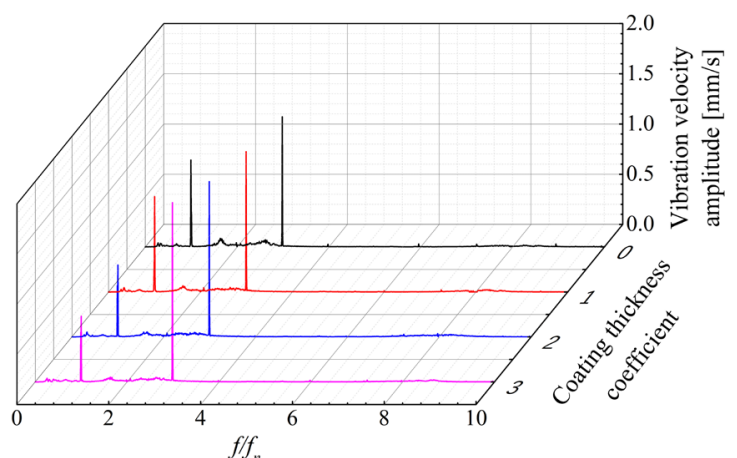

c)

$$
f / f_{n}
$$

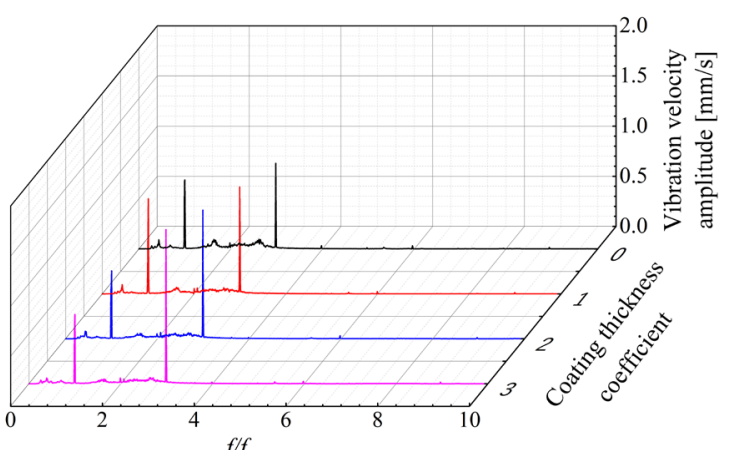

b)

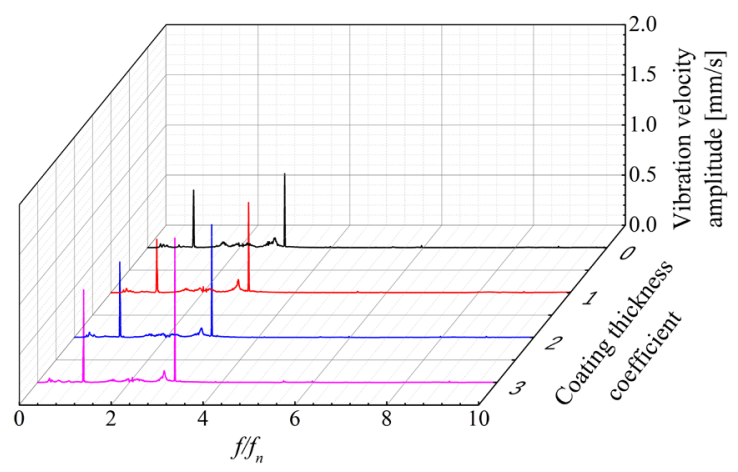

d)

Fig. 7. Frequency domain diagrams of vibration velocity at different measurement locations under clear water flow and design flow rate $Q_{d}$; at measurement locations: a) $M_{1}$; b) $M_{2}$; c) $M_{3}$; and d) $M_{4}$ 
mentioned above, the hydraulic efficiency $\eta_{h}$ under sediment flow is lower than that under clear water flow. As a result, the total efficiency $\eta$ under sediment flow is lower than that under clear water flow.

\subsection{Vibration Analysis}

Fig. 7 shows the frequency domain diagrams of vibration velocity at different measurement locations under clear water flow.

Based on the above figure, the axis rotating frequency (ARF) $\left(f_{n}\right)$ and blade passing frequency (BPF) (equal to 3 times $f_{n}$ ) are the main excitation frequencies of the pump vibration velocity at each measurement location. The vibration velocity amplitude at the frequency of $1 \mathrm{ARF}$ is the largest at every measurement location and coating thickness coefficient. It is also proportional to the coating thickness coefficient.

The vibration velocity amplitude at the frequency of $1 \mathrm{BPF}$ is just below that under the frequency of $1 \mathrm{ARF}$ at every measurement location and coating thickness coefficient. The vibration velocity amplitude at the frequency of $1 \mathrm{ARF}$ becomes the largest for the coating thickness coefficient $K_{1}$ at every measuring location except for location $M_{4}$.

Fig. 8 shows frequency domain diagrams of vibration velocity at different measurement locations under sediment-laden flow and design flow rate $Q_{d}$.

It can be seen that the characteristics of the vibration velocity at each measurement location under sediment-laden flow are the same as that under clear water flow.

A three-dimensional diagram of the relationship between the peak value of the vibration velocity amplitude and the coating thickness coefficient under design flow rate $Q_{d}$ is shown in Fig. 9.

The peak value of vibration velocity amplitude increases with the increase of the coating thickness coefficient under both sediment-laden flow and clear water flow. This is because of rotor-stator interaction between blade and volute tongue, and the thicker blade coating increases the liquid velocity at the blade outlet, which intensifies this kind of interaction. The thicker coating also means more rotating mass and more imbalance.

When the coating thickness coefficient is $K_{0}$, $K_{1}$ and $K_{2}$, the vibration velocity amplitude under sediment-laden flow is larger than under clear water
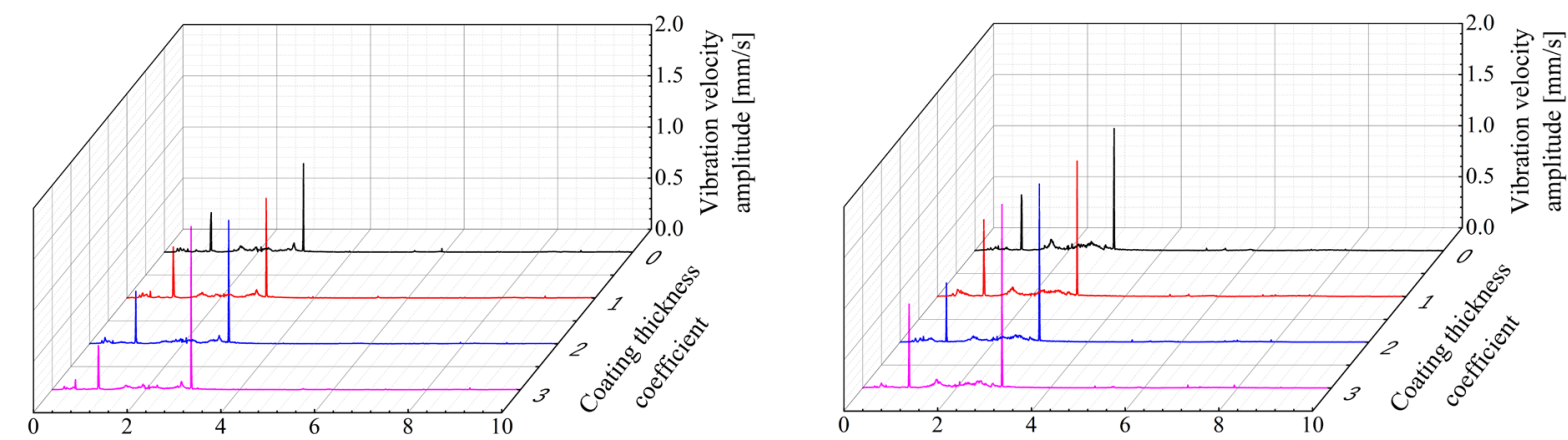

a)

$f / f_{n}$

b)

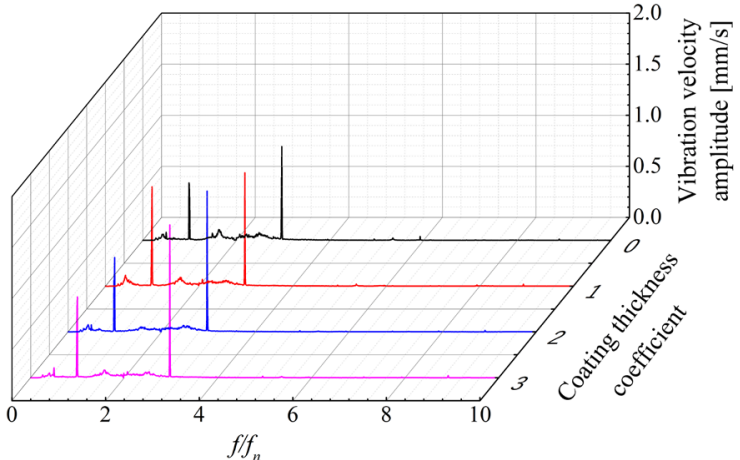

c)

Fig. 8. Frequency domain diagrams of vibration velocity under sediment-laden flow at measurement locations:

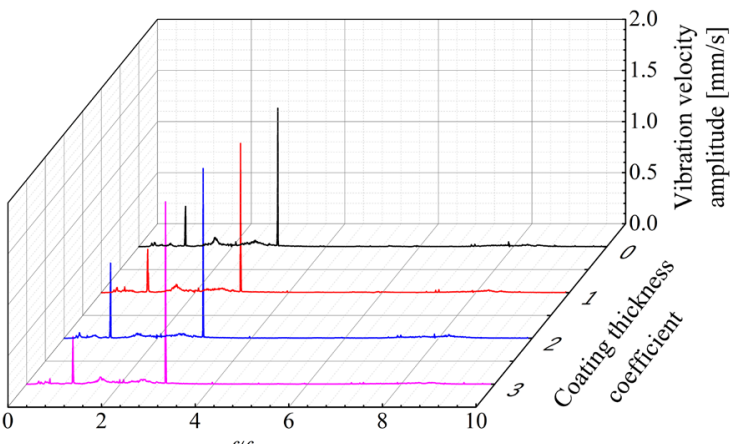

d) $\quad f / f_{n}$ a) $M_{1}$; b) $M_{2}$; c) $M_{3}$; and d) $M_{4}$ 


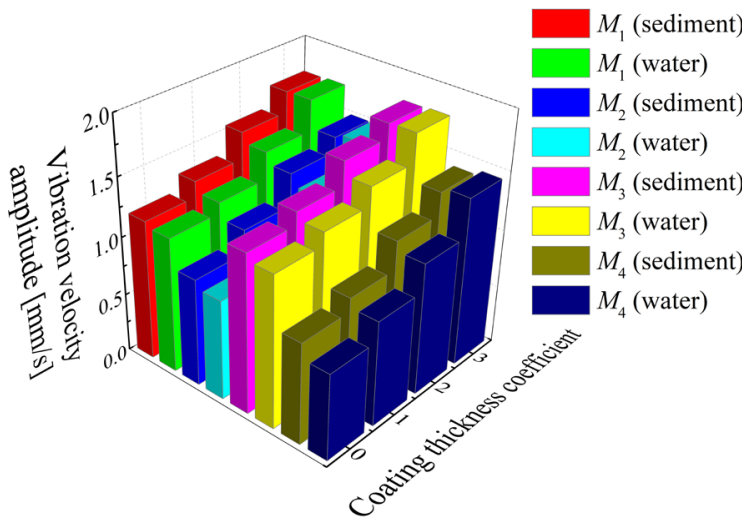

Fig. 9. Three-dimensional column of the amplitude for the velocity of the vibration

flow, but we see nearly no difference between them under the coating thickness coefficient $K_{3}$. The main reason is that the sediment particles of sediment-laden flow aggravate the instability or turbulence of the flow at volute tongue and a larger density of sediment-laden flow has a stronger interaction with volute. However, this kind of influence is covered by the negative effect of much large coating thickness coefficient on it. Therefore the vibration velocity amplitude does not show the difference between sediment-laden flow and water flow only on larger coating thickness coefficients.

\subsection{Pressure Fluctuations}

Fig. 10 shows the frequency domain diagrams of pressure fluctuations under different clear water flow rates and different coating thickness coefficients.

As shown in the above figure, the outlet pressure fluctuation features similar behaviour under different coating thickness coefficients and water flow rates. The ARF and BPF are the main excitation frequencies of the pump pressure fluctuation amplitude, and the pressure fluctuation at the frequency of $1 \mathrm{BPF}$ is significantly larger than that at the frequency of 1 ARF. At the same time, the difference of the pressure fluctuation at the frequency of $1 \mathrm{ARF}$ is not much different among experimental cases, which indicates that the effect and response of blade rotation on pressure fluctuation are more significant than axis rotation. Furthermore, under the same coating thickness coefficient, the peak pressure fluctuation under design flow rate $Q_{d}$ is lower than that under offdesign flow rates.

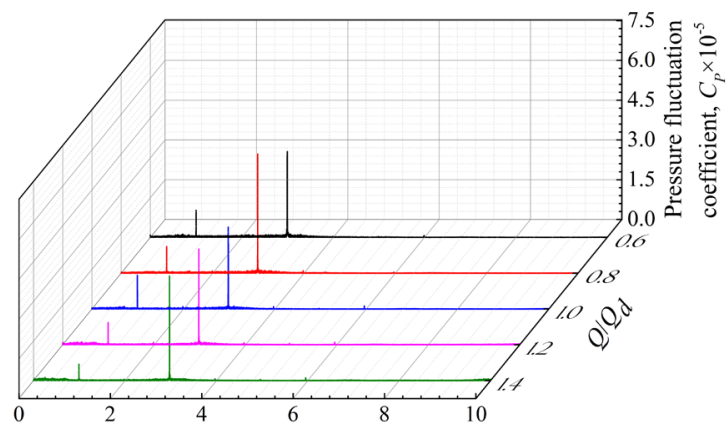

a)

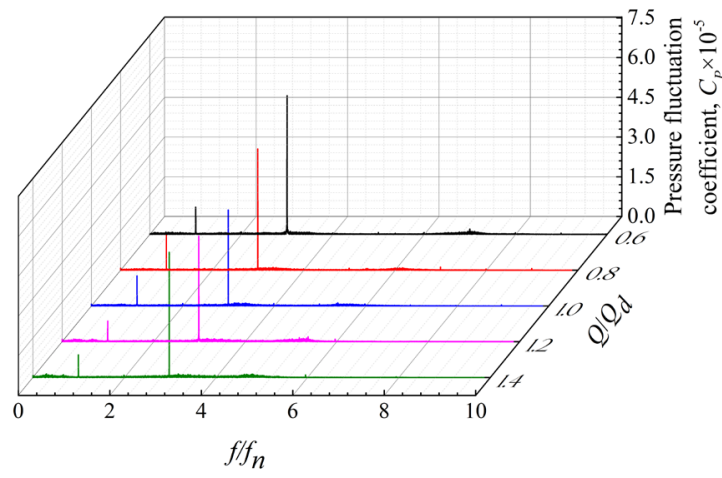

b)
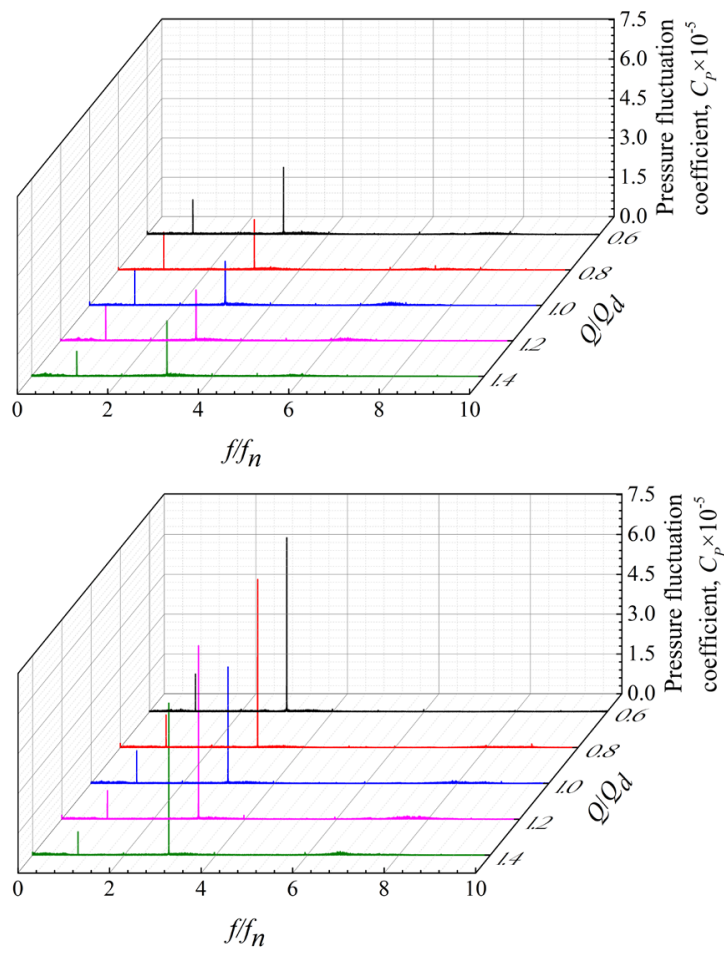

Fig. 10. Frequency domain diagrams of pressure fluctuation under different clear water flow rates at coating thickness coefficients:

a) $K_{1}$; b) $K_{2}$; c) $K_{3}$; and d) $K_{4}$ 


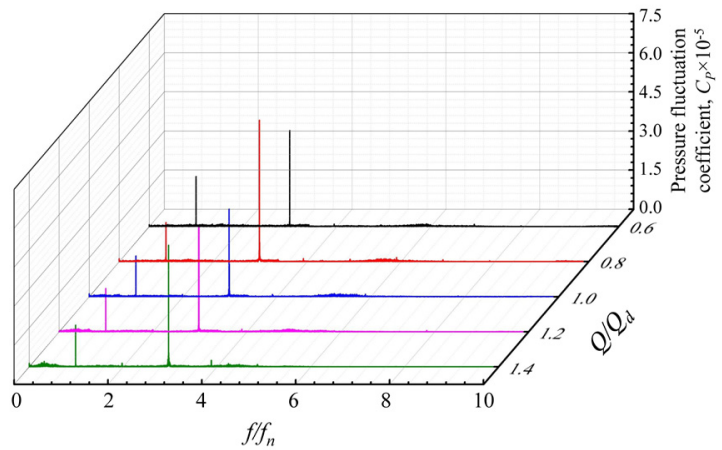

a)

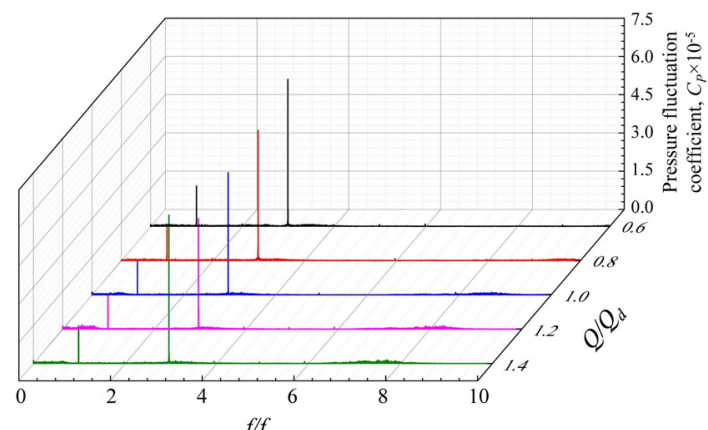

b)

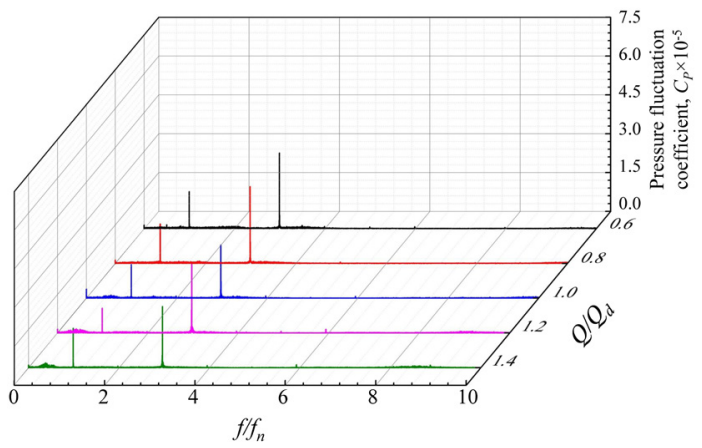

c)

$f / f_{n}$

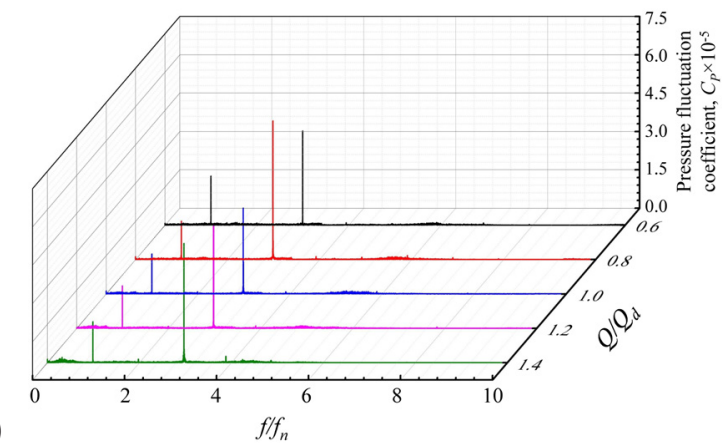

Fig. 11. Frequency domain diagrams of pressure fluctuation under different sediment-laden flow rates at coating thickness coefficients: a) $K_{1}$; b) $K_{2}$; c) $K_{3}$; and d) $K_{4}$

Fig. 11 shows the frequency domain diagrams of pressure fluctuation under different sediment-laden flow rates and different coating thickness coefficients. It can be seen that the characteristics of the pressure fluctuation under sediment-laden flow are basically the same as that under clear water flow.

Fig. 12 shows the line chart of peak pressure fluctuation.

As shown in the above figure, the variation trend of pressure fluctuation with increasing coating coefficient under both sediment-laden flow and clear water flow show similar behaviour.

Furthermore, under the same coating thickness coefficient, the peak value of pressure fluctuation under design flow rate $Q_{d}$ is lower than that under off-design flow rates. The peak values of pressure fluctuation under different flow rates increase first and then decrease with the increasing coating thickness coefficient, and the lowest points are all located at the coating thickness coefficient $K_{1}$. The peak values
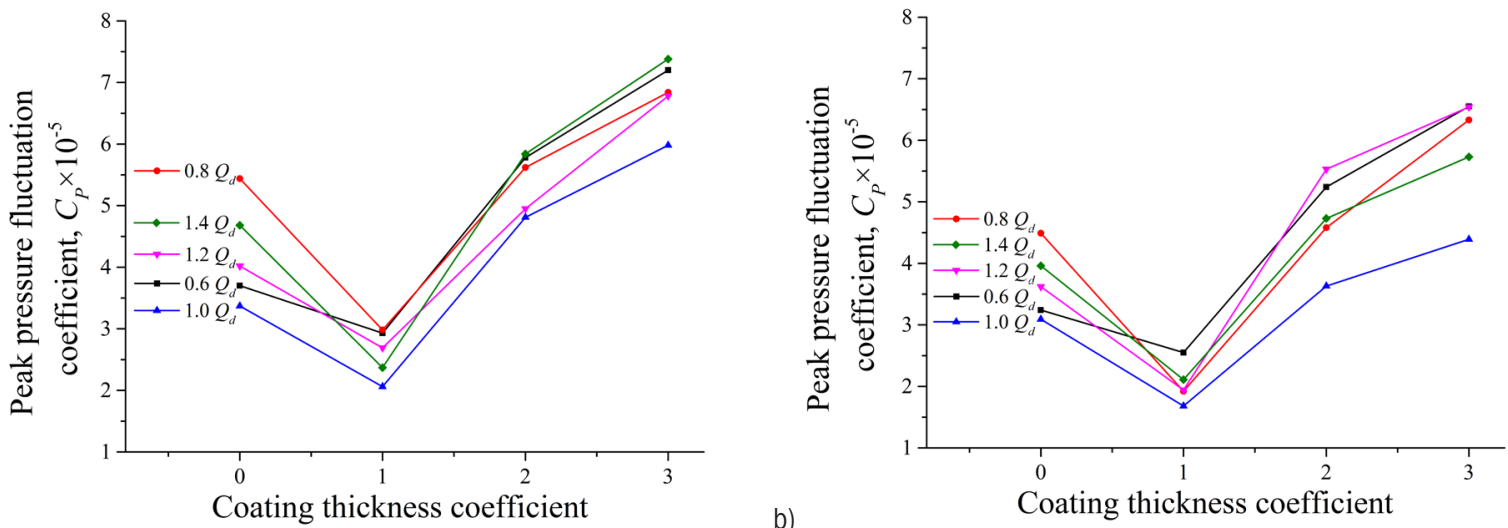

Fig. 12. Line chart of peak pressure fluctuation; a) under clear water flow; b) under sediment-laden flow 
of pressure fluctuation under sediment-laden flow are higher than that under clear water flow when the working conditions are the same.

\section{CONCLUSIONS}

In this paper, energy, vibration and pressure fluctuation of a centrifugal pump with different polyurethane coating thickness coefficients were experimentally studied under sediment-laden flow. The conclusions are as follows:

1. The head $H$ under both sediment-laden flow and clear water flow decreases significantly as the coating thickness coefficient increases. The head $H$ under sediment-laden flow is slightly larger than that under clear water flow under the same coating thickness coefficient when the volumetric flow rate is up to around $0.9 Q_{d}$. However, when the volumetric flow rate exceeds $0.9 Q_{d}$, the opposite is true. The efficiency $\eta$ gradually decreases with the increase of coating thickness coefficient for all flow rates, and the efficiency $\eta$ under sediment-laden flow is slightly smaller than that under clear water flow.

2. The ARF and BPF are the main excitation frequencies of the pump vibration velocity amplitude and outlet pressure fluctuation in all cases. The peak value of the vibration velocity amplitude and outlet pressure fluctuation at the frequency of $1 \mathrm{BPF}$ is the largest in all cases and that at the frequency of $1 \mathrm{ARF}$ is just the second.

3. The peak value of vibration velocity amplitude is also proportional to the coating thickness coefficient. When the coating thickness coefficients are $K_{0}, K_{1}$ and $K_{2}$, the peak value of vibration velocity amplitude under sedimentladen flow is larger than that under clear water flow, but there is a very small difference between them for coating thickness coefficient $K_{3}$.

4. The difference of the pressure fluctuation at the frequency of $1 \mathrm{ARF}$ varies little among different cases; however, that of $1 \mathrm{BRF}$ is pretty obvious. For the same coating thickness coefficient, the peak pressure fluctuation under design flow rate $Q_{d}$ is lower than that under off-design flow rates. The peak values of pressure fluctuation under different flow rates decrease first and then increase with the increasing coating thickness coefficient, and the lowest values are all found for the coating thickness coefficient $K_{1}$. The peak values of pressure fluctuation under sedimentladen flow are higher than that under clear water flow.

\section{ACKNOWLEDGEMENTS}

The authors would like to acknowledge the support given by the National Natural Science Foundation of China (51979126 and 51779106), and a project funded by the Priority Academic Program Development of Jiangsu Higher Education Institutions, Ministry of Education, Xihua University (szjj2016-068). The authors would like to thank the research program Energy Engineering P2-0401 funded by the Slovenian research agency ARRS.

\section{NOMENCLATURES}

$Q_{d}$ nominal flow rate, $\left[\mathrm{m}^{3} / \mathrm{h}\right]$

$Q \quad$ flow rate, $\left[\mathrm{m}^{3} / \mathrm{h}\right]$

$H$ pump head, [m]

$H_{t}$ theoretical head, [m]

$n$ rotational speed, [rpm]

$N$ sample number

$P \quad$ rated shaft power, $[\mathrm{kW}]$

$n_{s} \quad$ specific speeds

$\eta \quad$ efficiency, [\%]

$u_{1}$ circumferential velocity of impeller at blade inlet, $[\mathrm{m} / \mathrm{s}]$

$u_{2}$ circumferential velocity of impeller at blade outlet, $[\mathrm{m} / \mathrm{s}]$

$v_{u 1}$ circumferential velocity of flow at blade inlet, $[\mathrm{m} / \mathrm{s}]$

$v_{u 2}$ circumferential velocity of flow at blade outlet, $[\mathrm{m} / \mathrm{s}]$

$\varphi_{2} \quad$ extrusion coefficient at impeller outlet

$s_{u 2}$ thickness of blade at impeller outlet, [m]

$\gamma$ angle between section line on axial plane and streamline at impeller outlet, $\left[{ }^{\circ}\right]$

$h_{0} \quad$ slip coefficient

$D_{1}$ impeller inlet diameter, [mm]

$\mathrm{D}_{2}$ impeller outlet diameter, [mm]

$D_{3}$ inlet diameter of volute, [mm]

$D_{4}$ outlet diameters of volute, $[\mathrm{mm}]$

$b_{3}$ inlet width of volute, [mm]

$z$ number of blades

$\bar{p} \quad$ average static pressure, $[\mathrm{Pa}]$

$p_{i} \quad$ fluctuating static pressure, $[\mathrm{Pa}]$

$\delta \quad$ thickness of blade, [mm]

$\delta_{i} \quad$ thickness of blade coating, $[\mathrm{mm}]$

$\rho$ density, $\left[\mathrm{kg} / \mathrm{m}^{3}\right]$

$b_{2} \quad$ outlet impeller width, [mm]

$f_{n}$ axis rotating frequency, $[\mathrm{Hz}]$

$K_{i}$ coating thickness coefficient,

$C_{p}$ pressure fluctuation coefficient 


\section{REFERENCES}

[1] Wang, Z.-Y., Qian, Z.-D. (2017). Effects of concentration and size of silt particles on the performance of a double-suction centrifugal pump. Energy, vol. 123, p. 36-46, D0l:10.1016/j. energy.2017.01.142.

[2] Jin, W. (2014). Vibration Characteristics Analysis of Centrifugal Solid-Liquid Two-Phase Fluids Impeller. MSc thesis, Liaoning Technical University, Liaoning.

[3] Rodriguez, C.G., Egusquiza, E., Santos, I.F. (2007). Frequencies in the vibration induced by the rotor stator interaction in a centrifugal pump turbine. Journal of Fluids Engineering, vol. 129, no. 11, p. 1428-1435, Dol:10.1115/1.2786489.

[4] Han, W., Yi, J., Ren, N.-L. Yue, T., Quan, H. (2015). Effects of particles on pressure fluctuation characteristics in centrifugal pump. Journal of Drainage and Irrigation Machinery Engineering, vol. 33, no. 6, p. 467-474, D0I:10.3969/j. issn.1674-8530.14.1002. (in Chinese)

[5] Kumar, S., Gandhi, B.K., Mohapatra, S.K. (2014), Performance characteristics of centrifugal slurry pump with multi-sized particulate bottom and fly ash mixtures. Particulate Science and Technology, vol. 32, no. 5, p. 466-476, Dol:10.1080/027 26351.2014.894163.

[6] Zhao, B.-J, Yuan, S.-Q., Liu, H.-L., Huang, Z.-F., Tan, M.-G. (2008). Simulation of solid-liquid two-phase turbulent flow in double-channel pump based on mixture model. Transactions of the Chinese Society of Agricultural Engineering, vol. 24, no. 1, p. 7-12.

[7] Hazra, S.B., Steiner, K. (2007). Computation of dilute twophase flow in a pump. Journal of Computational and Applied Mathematics, vol. 203, no. 2, p. 444-460, D0l:10.1016/j. cam.2006.04.011.

[8] Zhang, Y., Li, Y., Cui, B., Zhu, Z.-H., Dou, H.-S. (2013). Numerical simulation, analysis of solid-liquid two-phase flow in centrifugal pump. Chinese Journal of Mechanical Engineering, vol. 26, p. 53-60, D0I:10.3901/CJME.2013.01.053.

[9] Tse, P.W., Wang, D. (2015). Enhancing the abilities in assessing slurry pumps' performance degradation, estimating their remaining useful lives by using captured vibration signals. Journal of Vibration and Control, vol. 23, no. 12, p. 1925-1937, D0I:10.1177/1077546315604522.

[10] Golru, S.S., Attar, M.M., Ramezanzadeh, B. (2014). Studying the influence of nano-Al203 particles on the corrosion performance and hydrolytic degradation resistance of an epoxy/polyamide coating on AA-1050. Progress in Organic Coatings, vol. 77, no. 9, p. 1391-1399, D0l:10.1016/j. porgcoat.2014.04.017.

[11] Chairman, C.A., Kumaresh-Babu, S.P. (2013). Mechanical and abrasive wear behavior of glass and basalt fabric-reinforced epoxy composites. Journal of Applied Polymer Science, vol. 130, p. 120-130, D0l:10.1002/app.39154.

[12] Jin, J., Rafiq, R., Gill, Y.Q., Song, M. (2013). Preparation and characterization of high performance of graphene/nylon nanocomposites. European Polymer Journal, vol. 49, no. 9, p. 2617-2626, D0I:10.1016/j.eurpolymj.2013.06.004.
[13] Kim, B.K., Lee, J.C. (1996). Waterborne polyurethanes and their properties. Journal of Polymer Science Part A: Polymer Chemistry, vol. 34, no. 6, p. 1095-1104, D0I:10.1002/(SICI)1099-0518(19960430)34:6<1095::AIDPOLA19>3.0.C0;2-2.

[14] Fu, J.J., Chen, T., Wang, M.D., Yang, N.W, Li, S.N, Wang, Y., Liu, X.D. (2013). Acid and alkaline dual stimuli-responsive mechanized hollow mesoporous silica nanoparticles as smart nanocontainers for intelligent anticorrosion coatings. ACS Nano, vol. 7, no. 12, p. 11397-11408, Dol:10.1021/ nn4053233.

[15] Luo, K.-K., Wang, Y., Liu, H.-L., Dular, M., Chen, J. Zhang, Z.-L. (2019). Effect of coating thickness on a solid-liquid two-phase flow centrifugal pump under water medium. Strojniški vestnik Journal of Mechanical Engineering, vol. 65, no. 4, p. 251-261, DOI:10.5545/sv-jme.2018.5865.

[16] Serrano, R.O.P., Santos L.P., Viana, E.M.F., Pinto, M.A., Martinez, C,B. (2018). Case study: Effects of sediment concentration on the wear of fluvial water pump impellers on Brazil's Acre river. Wear, vol. 408-409, p. 131-137, DOl:10.1016/j.wear.2018.04.018.

[17] Tarodiya, R., Gandhi, B.K. (2017). Hydraulic performance and erosive wear of centrifugal slurry pumps - a review. Powder Technology, vol. 305, p. 27-38, D0l:10.1016/j. powtec.2016.09.048.

[18] Walker, C.I., Robbie, P. (2013). Comparison of some laboratory wear tests and field wear in slurry pumps. Wear, vol. 302, no. 1-2, p. 1026-1034, D0l:10.1016/j.wear.2012.11.053.

[19] Zhang, R.-Z. (2014). Abrasion resistance analysis of spray polyurethane elastic coating on turbine blades. Surface Technology, vol. 43, no. 1, p. 11-15, Dol:10.16490/j.cnki. issn.1001-3660.2014.01.014.

[20] Chen, J. (2018). Effect of Coating on Operating Characteristics of Solid-Liquid Two-Phase Flow Centrifugal Pumps. MSc thesis, Jiangsu University, Jiangsu.

[21] Zhou, R., Lu, D.H., Jiang, Y.H., Li, Q.N. (2005). Mechanical properties and erosion wear resistance of polyurethane matrix composites. Wear, vol. 259, no. 1-6, p. 676-683, D0l:10.1016/j.wear.2005.02.118.

[22] Zhong, P., Peng, E.-G., Li, J., Xiao, X. (2007). Study of erosion behaviour of polyurethane-urea coating. Tribology, vol. 27, no. 5, p. 447-450, DOI:10.3321/j.issn:1004-0595.2007.05.010. (in Chinese)

[23] Sun, J.-H, Chai, Y., Wang, G.-L. (2010). Review on effects of sediment on the water quality of the Yellow River. Journal of Sediment Research, vol. 1, p. 72-80, Dol:CNKI:SUN:NS YJ.0.2010-01-012. (in Chinese)

[24] Liu, H. L., Tan, M.-G, Yuan, S.-Q. (2006). Research on calculation of theoretical head of centrifugal pumps. Transactions of the Chinese Society for Agricultural Machinery, vol. 37, no. 12, p. 87-90, DOI:CNKI:SUN:NYJX.0.2006-12-021. (in Chinese)

[25] He, X.-J., Lao, X.-S. (2009). Review of formulas for efficiency calculation of centrifugal pump. Pump technology, vol. 6, p. 24-27, DOI:CNKI:SUN:SBJS.0.2009-06-007. (in Chinese) 